\title{
A Improved Particle Swarm Optimization Algorithm with Dynamic Acceleration Coefficients
}

\author{
Gang Ma*, Renbin Gong, Qun Li, Gang Yao \\ PetroChina Research Institute of Petroleum Exploration \& Development-Northwest, \\ Lanzhou 730020, Gansu Province, China \\ ${ }^{*}$ Corresponding author, e-mail: magangzh@126.com
}

\begin{abstract}
Particle swarm optimization (PSO) is one of the famous heuristic methods. However, this method may suffer to trap at local minima especially for multimodal problem. This paper proposes a modified particle swarm optimization with dynamic acceleration coefficients (ACPSO). To efficiently control the local search and convergence to the global optimum solution, dynamic acceleration coefficients are introduced to PSO. To improve the solution quality and robustness of PSO algorithm, a new best mutation method is proposed to enhance the diversity of particle swarm and avoid premature convergence. The effectiveness of ACPSO algorithm is tested on different benchmarks. Simulation results found that the proposed ACPSO algorithm has good solution quality and more robust than other methods reported in previous work.
\end{abstract}

Keywords: PSO, dynamic acceleration coefficients, heuristic

\section{Introduction}

Parcicle swarm optimization (PSO) is a population-based search optimization technique introduced by Kennedy and Eberhart in 1995 [1] [2]. On the simulation of the behavior of bird flocking, the particle swarm algorithm starts with the random initialization of a population of individuals in the search space [1]. It tries to find the global best solution by adjusting the trajectory of each individual toward its own best location and the best particle of the whole swarm at each iteration [1]. The PSO method is becoming very popular due to its simplicity of implementation and quick convergence to a global optima. Since PSO was introduced, it has become a popular optimizer and has widely been applied in practical problems. Syahputra [3] uses PSO Algorithm to optimize configuration of distribution network with distributed energy resources integration. Lu [4] introduces PSO algorithm into the Artificial Neural Network training and construct a BP neural network model to improve the prediction accuracy for the cost forecasting of transmission line project based on historical project data. Wang [5] introduced PSO into the hardware structure design of the dynamic compensator to facilitate the application of an optimized compensator to real-time online measurement.

In the particle swarm algorithm, the trajectory of each individual in the search space is adjusted by dynamically altering the velocity of each particle, according to its own flying experi ence and the flying experience of the other particles in the search space. In a $D$-dimensional space, a particle represents a potential solution presented by their velocity and position and $D$ is the number of the parameters. During a search process, each particle is attracted by its previous best particle (pbest) and the global best particle (gbest) as follows.

$$
\begin{aligned}
& v_{i d}(t+1)=w \cdot v_{i d}(t)+c 1 \cdot r 1 \cdot\left(\text { pbest }_{i d}(t)-x_{i d}(t)\right)+c 2 \cdot r 2 \cdot\left(\text { gbest }_{d}(t)-x_{i d}(t)\right) \\
& x_{i d}(t+1)=x_{i d}(t)+v_{i d}(t+1)
\end{aligned}
$$

Where $i=1,2, \ldots, N$ is the particle's index, $N$ is the population size, $X_{i}=\left(x_{i 1}, x_{i 2}, \ldots, x_{i D}\right)$ is the position of the $i$ th particle. $V_{i}=\left(v_{i 1}, v_{i 2}, \ldots, v_{i D}\right)$ represents the velocity of the $i$ th particle; the pbest $_{i}=\left(\right.$ pbest $_{1}$, pbest $_{2}, \ldots$, pbest $\left._{D}\right)$ is the best previous position in the $i$ th particle own history and the gbest $=\left(\right.$ gbest $_{1}$, gbest $_{2}, \ldots$, gbest $\left._{D}\right)$ is the global best particle found by all particle so far. 
The inertia weight $w$ was introduced by Shi and Eberhart [6] [7] for the classical PSO, which is used to balance the exploration and the exploitation abilities, and suggested that the optimal solution can be improved by altering the value of $w$ linearly decreasing (PSO- $w$ ) from 0.9 to 0.4 . In general, the higher values of $w$ help particles to search space more thoroughly in the process and find the global minima, while lower values help to search the local space and find the local minima.

$$
w=\left(w_{\max }-w_{\min }\right) \cdot \frac{\text { MAXITER-iter }}{M A X I T E R}+w_{\min }
$$

Jiao [8] made an improved method on the inertia weight and proposed the dynamic inertia weight decreases according to iterative generation increasing as below.

$$
w=w \cdot u^{-k},(w \epsilon[0,1], u \epsilon[1.001,1.005])
$$

The $r 1$ and $r 2$ are two uniform random numbers generated independently within the range of $[0,1], c 1$ and $c 2$ are two learning factors which control the influence of the social and cognitive components, and $t=1,2, \ldots, F E s$ indicates the iteration number. The first part of (1) represents the previous velocity, which keeps its own momentum for particles to roam across the search space. The second part, known as the cognitive component, represents the personal judging of each particle that helps the particle to move toward its own best position found so far. In the past decades, many variants of PSO have been proposed [6] [9] [10].

\section{Research Method}

In this paper, we propose a dynamic parameter adjusting strategy and a neighborhood learning strategy. The major objective of this development is to improve the performance after a predefined number of generations, through empirical simulations with well-known benchmarks.

\subsection{Parameter Dynamically Adjusting Strategy}

Although the PSO-w [6] can find a satisfactory solution with fast speed, its ability to find optimum solution is limited, because of the diversity loss at the latter stage of evolution process. To find the optimum solution [1], it is better to encourage the individuals to search through the entire search space during the early part of the search, avoiding clustering around local optima, and during the later stage, convergence towards the global optima is encouraged. Figure 1 shows the curve of $c 1$ and $c 2$ trend.

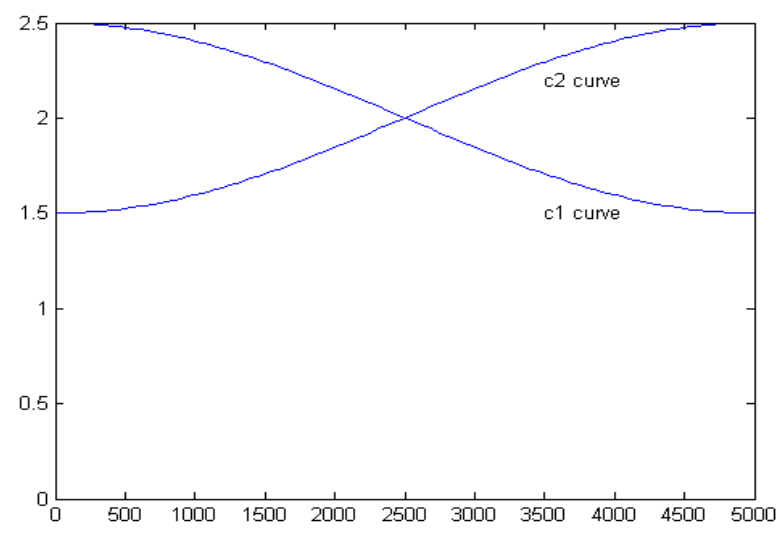

Figure 1. The curve of $c 1$ and $c 2$ trend

The parameters affects the performance of PSO in the optimum solutions. Under this new development, we reduce social component and increase the cognitive component, by dynamiclly changing the acceleration coefficients. With a large social component and small 
cognitive component at the beginning, particles are allowed to move around the search space, instead of moving toward the local optimum. On the other hand, a small social component and a large cognitive component allow the particles to converge to the global optima in the latter part of the optimization. This modification can be mathematically represented as follows:

$$
\begin{aligned}
& c 1=2.0+0.5 \cdot \cos \frac{\pi \cdot i t e r}{M A X I T E R} \\
& c 2=2.0-0.5 \cdot \cos \frac{\pi \cdot i t e r}{M A X I T E R}
\end{aligned}
$$

\subsection{Swarm Diversity Enhanced Method}

In the basic PSO algorithm, particles are attracted by their pbests and the gbest and all particles move quickly to the same direction and the loss of swarm diversity is a serious problem for PSO algorithms. To maintain diversity of a swarm, some excellent strategies have been proposed. Riget [9] proposed a diversity-guided PSO, which defines a repulsion phase based on a new velocity updating mechanism and calculating diversity of the swarm is necessary. Sun et al. [10] introduced a mutation operator to enhance the swarm diversity. When the diversity is less than a predefined value, a mutation on gbest is conducted to increase the dissimilarities among particles. The method has good performance but it costs much computational time to calculate the swarm diversity.

In order to maintain diversity and avoid calculating the diversity, this paper proposes a novel mechanism instead of diversity computing for PSO. We add a third part which represents the collaborative effect of the particles, to find the global optima.

$$
\begin{gathered}
v_{i d}(t+1)=w \cdot v_{i d}(t)+c 1 \cdot r 1 \cdot\left(\text { pbest }_{i d}(t)-x_{i d}(t)\right)+c 2 \cdot r 2 \cdot\left(\text { gbest }_{d}(t)-x_{i d}(t)\right) \\
+(c 3+c 4) \cdot r 3 \cdot\left(\text { pbest }_{j d}(t)-x_{i d}(t)\right)
\end{gathered}
$$

The gbest $(t)$ does not change for a constant times which means that the particles find the local optima and the diversity loss of the swarm is a problem. When this happens, we can set $c 3=0.1$ to enhance the mutation for each particle. When pbest $t_{i}$ does not change for a constant times which means that the particle has been trapped around the local optima and we can set $c 4=0.1$ to enhance the mutation for the particle. The pbest $_{j d}(t)$ is best position for the particle $j$ found so far and $r 3$ is a random number in $[0,1]$.

\subsection{Proposed PSO}

The flowchart of the ACPSO algorithm is shown in Figure 2. The stop criteria are that the maximum iteration number is reached or the minimum error condition is satisfied. In ACPSO algorithm, each particle of the swarm dynamically changes acceleration coefficients and execute diversity improved method during the search process. 


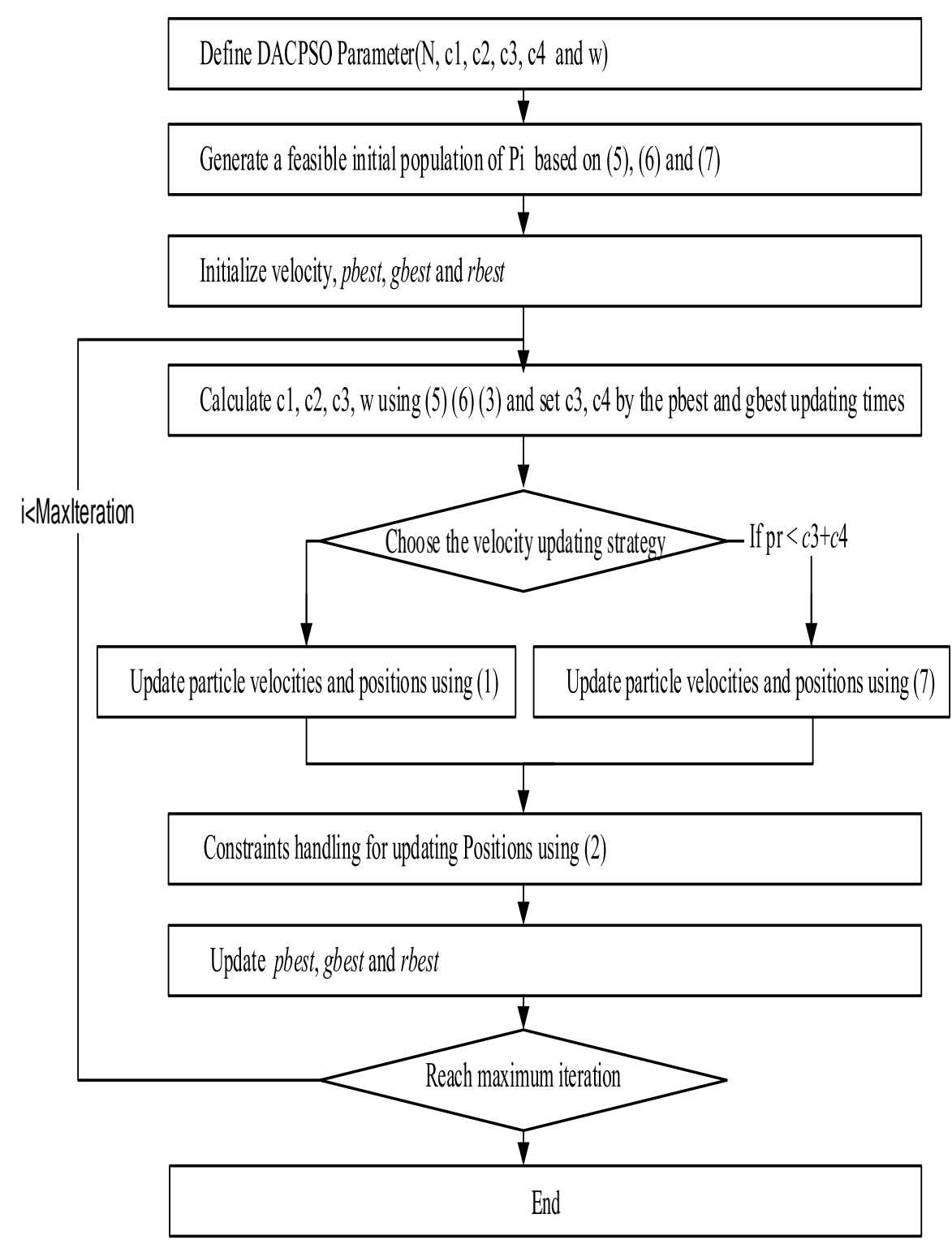

Figure 2. Flowchart of proposed ACPSO algorithm

\section{Results and Analysis}

The functions with dimension 30 is used to show the characteristics of the proposed PSO. The parameters are set as below. Table 1 illustrates the functions, including the best fitness value and average velocity of all particles. Table 2 illustrates the variation of parameters in the evolution, including inertia weight, $c 1$, and $c 2$.

Table 1. Benchmark Problems Tested in the Experiments

\begin{tabular}{cccc}
\hline Problems & Name & $\mathrm{X}$ & $\mathrm{f}\left(\mathrm{x}^{\mathrm{D}}\right)$ \\
\hline F1 & Rosenbrock's function & {$[-2.048,-2.048]$} & 0 \\
F2 & Ackley's function & {$[-32.768,32.768]$} & 0 \\
F3 & Griewanks's function & {$[-600,600]$} & 0 \\
F4 & Rastrigin's function & {$[-5.12,5.12]$} & 0 \\
F5 & Nocontinuous Rastrigin's function & {$[-5.12,5.12]$} & 0 \\
F6 & Schewfel function & {$[-500,-500]$} & 0 \\
F7 & Weierstrass function & {$[-0.5,-0.5]$} & 0 \\
F8 & E_ScafferF6 function & {$[-100,100]$} & 0 \\
\hline
\end{tabular}


Table 2. PSO Algorithms for Comparison

\begin{tabular}{ccc}
\hline Algorithm & Parameters & References \\
\hline SPSO & $w: 0.729, c 1=c 2=1.49445$ & {$[1]$} \\
PSO- $w$ & $w: 0.9-0.4, c 1=c 2=2$ & {$[6]$} \\
IPSO & $w_{\text {initial }}: 0.3, u=1.002, c 1=c 2=1.49445$ & {$[8]$} \\
ACPSO & w: $0.9-0.4, c 1_{\text {initial }}=2, c 1_{\text {initial }}=1$ & This paper \\
\hline
\end{tabular}

The result shown in Table 3 is typical. There are 8 functions tested and six of them show the best result. Mostly the best fitness value of ACPSO is better than that of others. And the Figuire 3-8 show the fitness trend of the search process and ACPSO can always find a better solution. It can be known that PSO algorithm quite depends on the inertia weight in largescale optimization problems and the performance of standard PSO can be improved by the inertia weight of particle swarm changing along with the nonlinear ideal inertia weight. As the the parameter $c 1$ starts with a large value and the particle can keep its direction and try to find more space and at the last stage $c 1$ gets smaller and smaller and but the $c 2$ get bigger as a result the particle try to search the same direction and and finally improve the searching result which can be shown in Figure 3-8.

Table 3. Comparison of Results on 50-time Independent Random Testing and $D=30$

\begin{tabular}{|c|c|c|c|c|}
\hline Problems & SPSO & PSO-w & IPSO & ACPSO \\
\hline Prodiems & mean/std & mean/std & mean/std & mean/std \\
\hline F1 & $3.24 e+1 / 4.99 e+2$ & $2.82 e+1 / 2.412$ & $5.72 e+1 / 1.41 e+3$ & $2.74 e+1 / 1.27$ \\
\hline $\mathrm{F} 2$ & $2.31 / 5.78 \mathrm{e}-1$ & $2.18 / 04.14 \mathrm{e}-1$ & $7.38 / 1.93$ & $5.87 e-1 / 3.96 e-1$ \\
\hline F3 & $1.45 e-2 / 2.87 e-4$ & $1.55 e-2 / 5.15 e-4$ & $2.57 e-1 / 4.55 e-2$ & $1.24 e-1 / 1.32 e-2$ \\
\hline $\mathrm{F} 4$ & $7.92 e+1 / 4.43 e+2$ & $72.167 / 4.384 \mathrm{e}+2$ & $68.822 / 2.95 e+2$ & $52.29 / 4.75 e+2$ \\
\hline F5 & $79.90 / 5.25 e+02$ & $77.17 / 7.14 \mathrm{e}+2$ & $88.27 / 6.69 e+2$ & $74.87 / 3.67 e+2$ \\
\hline F6 & $3.60 e+3 / 4.97 e+5$ & $2.74 e+03 / 5.58 e+05$ & $5.95 e+3 / 5.10 e+5$ & $3.07 e+3 / 4.10 e+5$ \\
\hline F7 & $9.95 / 8.71$ & $10.25 / 5.51$ & $17.14 / 5.34$ & $1.59 / 1.43$ \\
\hline F8 & $9.69 / 0.73$ & 10.89/ 0.63 & $11.17 / 1.70$ & $8.99 / 1.27$ \\
\hline
\end{tabular}

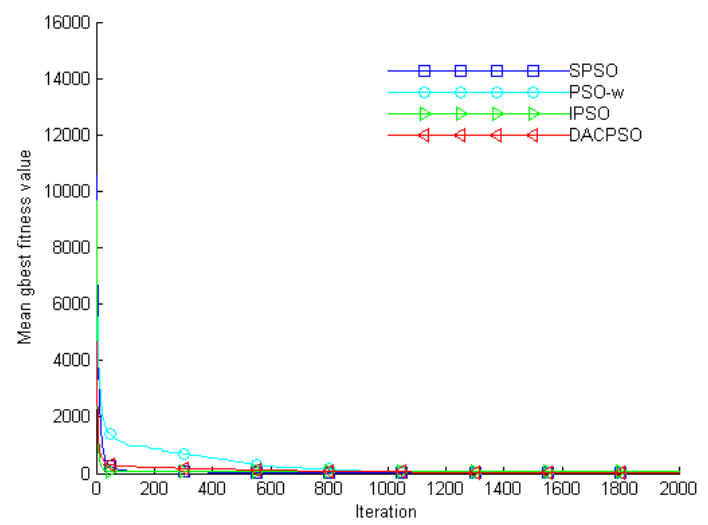

Figure 3. Rosenbrock's function

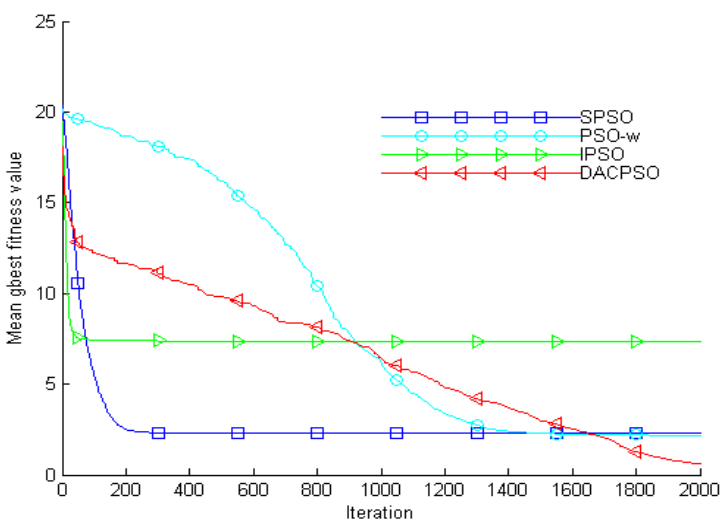

Figure 4. Ackley's function 


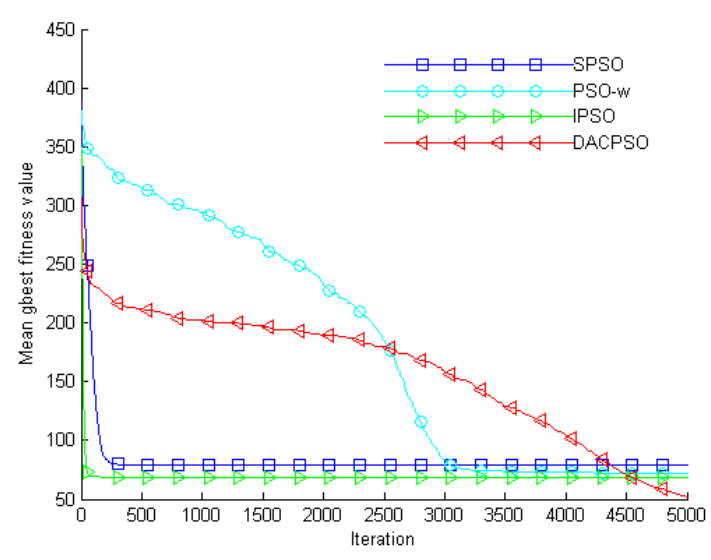

Figure 5. Rastrigin's function

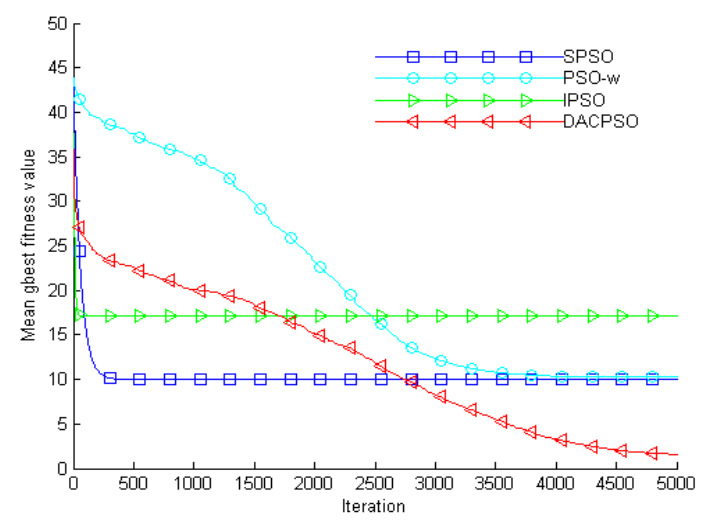

Figure 7. Weierstrass function

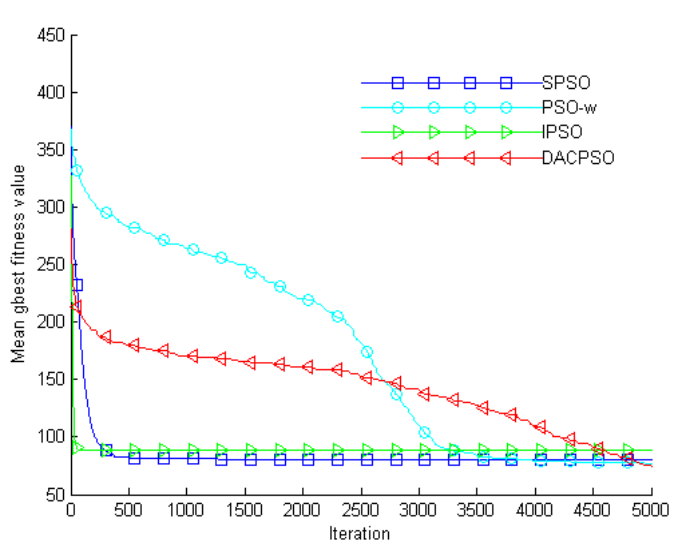

Figure 6. Nocontinuous Rastrigin's function

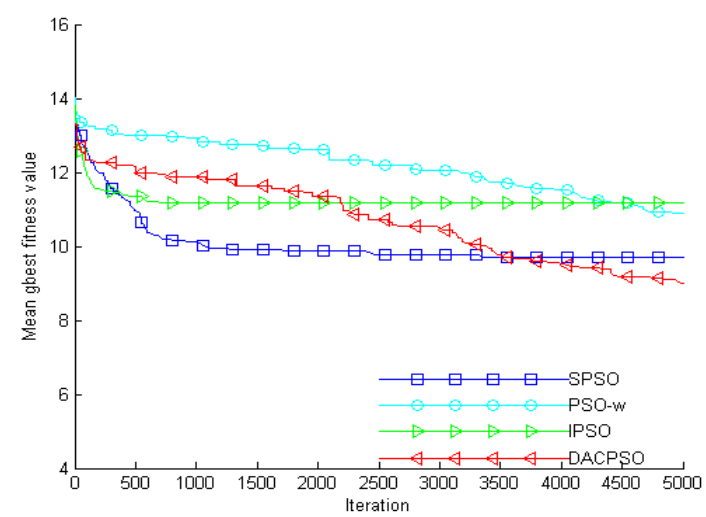

Figure 8. E_ScafferF6 function

\section{Conclusion}

This paper presents an improved PSO algorithm called ACPSO to solve complex optimization problems. The proposed approach employs two strategies including dynamic acceleration coefficients strategy and diversity improved method. By combining the two strategies, ACPSO achieves a trade-off between the exploration and exploitation abilities. To verify the performance of ACPSO, different benchmark functions are tested in the experiments. From the experiments and analysis we observe that this strategy enables our algorithm to perform more efficiently.

\section{References}

[1] R Eberhart, J Kennedy. A New Optimizer Using Particle Swarm Theory. Proceedings of the 6th International Symposi. Micro Machine \& Human Science. Nagoya. 1995: 39-43.

[2] J Kennedy, R Eberhart. Particle swarm optimization. IEEE International Conference on Neural Networks. Piscataway, NJ. 1995; 4:1942-1948.

[3] R Syahputra, I Robandi, M Ashari. Reconfiguration of Distribution Network with Distributed Energy Resources Integration Using PSO Algorithm. TELKOMNIKA (Telecommunication, Computing, Electronics and Control). 2015; 13(3): 759-766.

[4] Y Lu, D Niu, B Li M Yu. Cost Forecasting Model of Transmission Project based on the PSO-BP Method. TELKOMNIKA (Telecommunication, Computing, Electronics and Control). 2014; 12(4): 773-778.

[5] W Wang, Z Zhang. Online Correction of the Dynamic Errors in a Stored Overpressure Measurement System. TELKOMNIKA (Telecommunication, Computing, Electronics and Control). 2015; 13(3): 828-835. 
[6] Y Shi, RC Eberhart. A modified particle swarm optimizer. Proceedings of the IEEE Congress on Evolutionary Computation. Piscataway, NJ. 1998:69-73.

[7] Y Shi, RC Eberhart. Empirical study of particle swarm optimization. Proceedings of the IEEE Congress on Evolutionary Computation. Piscataway, NJ. 1999:1945-1950.

[8] B Jiao, Z Lian, X Gu. A dynamic inertia weight particle swarm optimization algorithm. Chaos, Solitons \& Fractals. 2008; 37(3): 698-705.

[9] J Riget, JS Vesterstom. A diversity-guided particle swarm optimizer-the ARPSO. Technical report, EVAlife, Denmark. 2002.

[10] J Sun, BW Xu, W Fang. A diversity-guided quantum-behaved particle swarm optimization algorithm. International Conference on Simulated Evolution and Learning. 2006: 497-504. 